\title{
Clinical Comparison of Influenza A and B Virus Infection in Hospitalized Children
}

Sang Hyuk $\mathrm{Ma}^{2}$ Seungwon Jung ${ }^{1}$, Joon Hee Lee ${ }^{1}$, Jin Han Kang ${ }^{1}$, Hak Sung Lee2, Jae Won Choi ${ }^{2}$, Jaywon Lee ${ }^{3}$

1. Department of Pediatrics, Seoul St. Mary's Hospital, College of Medicine, The Catholic University of Korea, Seoul, 2.Department of Pediatrics, Changwon Fatima Hospital, Changwon,

3. Korea Advanced Institute of Science and Technology, Seoul, the Republic of Korea

\section{Purpose: The objective of this} study was to compare the clinical characteristics of influenza $A$ and $B$ infections and analyze the effect of oseltamivir in hospitalized children.

Methods: We investigated children under the age of 15 , who were diagnosed with influenza $A / H 1 N 1$, A/H3N2, or B from January to April 2014. The subjects were admitted to the Changwon Fatima Hospital and diagnosed using a rapid antigen test from nasopharyngeal swabs. The medical records of the patients were retrospectively reviewed.
Results: A total of 302 pediatric patients with influenza were enrolled. Influenza B infection was the most common type ( $n=187,61.9 \%)$, followed by $\mathrm{A} / \mathrm{H} 3 \mathrm{~N} 2(\mathrm{n}=100,33.1 \%)$ and $\mathrm{A} / \mathrm{H} 1 \mathrm{~N}$ $(n=15,5.0 \%)$. Compared to patients diagnosed with influenza $A$, patients diagnosed with influenza $B$ were older $(P=0.005)$, and the duration of fever was significantly longer $(P=0.001)$. A total of 161 patients $(53.3 \%$ ) had been vaccinated against influenza during the season, before admission. Among the patients infected with $\mathrm{A} / \mathrm{H} 3 \mathrm{~N} 2$ and $\mathrm{B}$, the duration of fever was shorter in oseltamivir recipients compared to oseltamivir non-recipients $(\mathrm{P}=0.026$ and $\mathrm{P}=0.004$, respectively).

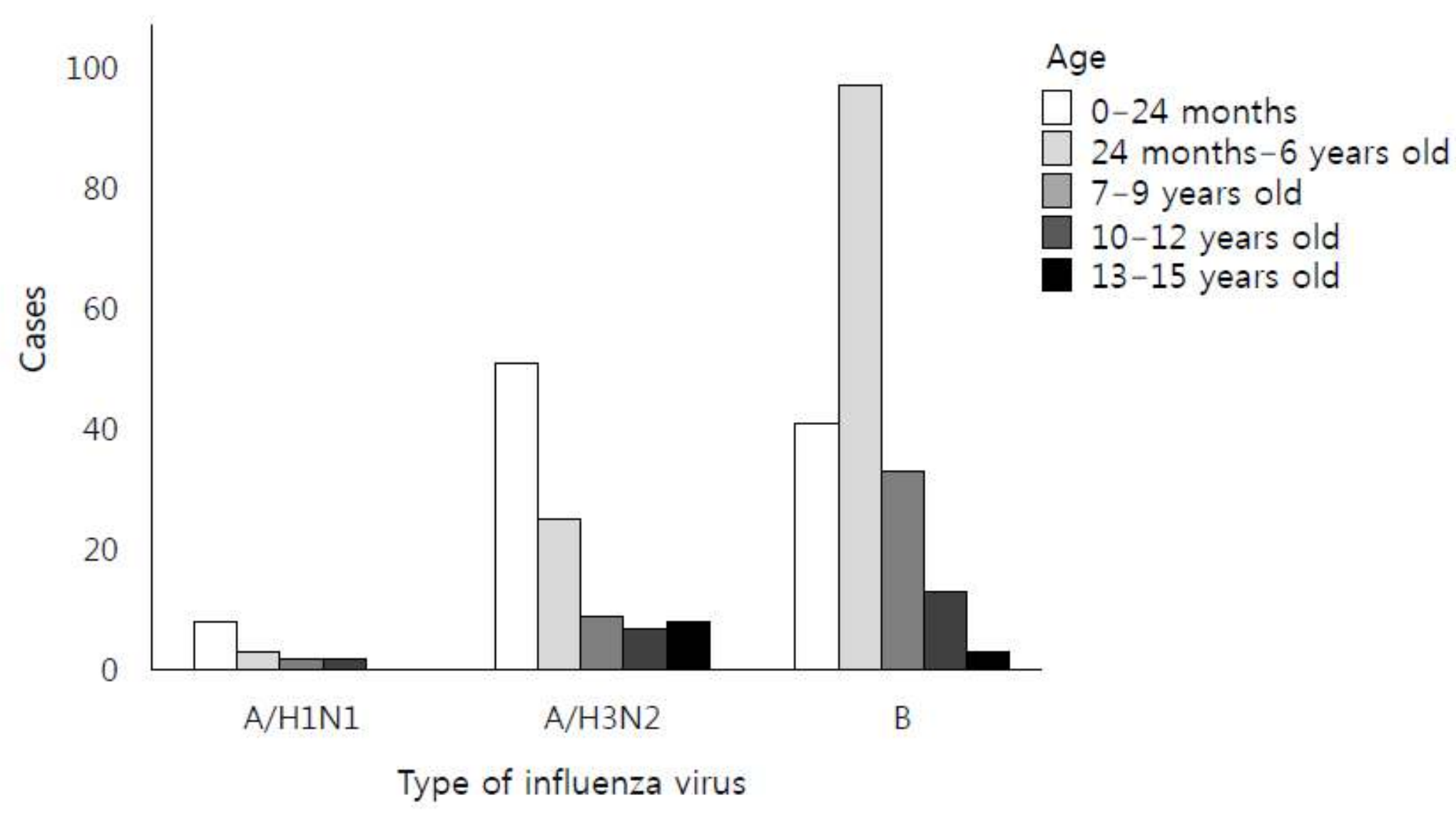

Fig. 1. The number of children according to the age group.
Table 1. Clinical and Laboratory Characteristics according to Type of Influenza

\begin{tabular}{|c|c|c|c|c|}
\hline Type of influenza & A/H1N1 $(n=15)$ & A/H3N2 $(n=100)$ & $B(n=187)$ & $P$-value \\
\hline Duration of admission (day) & $2.6 \pm 1.0$ & $2.8 \pm 1.1$ & $3.4 \pm 1.3$ & $<0.001$ \\
\hline Duration of fever (day) & $3.9 \pm 1.4$ & $3.5 \pm 1.4$ & $4.8 \pm 1.6$ & 0.001 \\
\hline Duration of fever after oseltamivir administration (day)* & $1.7 \pm 0.9^{*}$ & $1.8 \pm 0.8^{\star}$ & $2.2 \pm 1.1^{*}$ & 0.027 \\
\hline Duration of fever in non-oseltamivir recipients (day) ${ }^{\dagger}$ & - & $4.0 \pm 1.8^{\dagger}$ & $5.4 \pm 1.5^{\dagger}$ & 0.001 \\
\hline Fever & $15(100.0)$ & $100(100.0)$ & $186(99.5)$ & 0.735 \\
\hline Cough & $15(100.0)$ & $100(100.0)$ & $181(97.3)$ & 0.208 \\
\hline Coryza & $15(100.0)$ & $95(95.0)$ & $171(91.9)$ & 0.349 \\
\hline Diarrhea & $2(13.3)$ & $5(5.0)$ & $17(9.1)$ & 0.347 \\
\hline White blood cells $\left(/ \mathrm{mm}^{3}\right)^{*}$ & $6,631.3 \pm 2,233$ & $6,175 \pm 2,642$ & $5,998 \pm 2,947$ & 0.385 \\
\hline Platelet $\left(/ \mathrm{mm}^{3}\right)^{*}$ & $236,200 \pm 58,200$ & $221,700 \pm 73,700$ & $200,800 \pm 56,700$ & 0.112 \\
\hline Alanine transaminase (IU/L)* & $32.1 \pm 9.2$ & $31.3 \pm 13.0$ & $33.4 \pm 12.8$ & 0.261 \\
\hline Alanine aminotransferase (IU/L)* & $19.7 \pm 9.3$ & $106.9 \pm 15.3$ & $15.9 \pm 6.8$ & 0.167 \\
\hline C-reactive protein $(\mathrm{mg} / \mathrm{dl})^{*}$ & $0.97 \pm 1.03$ & $1.16 \pm 1.19$ & $0.81 \pm 1.11$ & 0.283 \\
\hline Infiltration on X-ray & $2(13.3)$ & $11(11.0)$ & $17(9.1)$ & 0.791 \\
\hline Vaccination against influenza & $5(33.3)$ & $54(54.0)$ & $102(54.5)$ & 0.552 \\
\hline Previous intrafamilial exposure & $6(40.0)$ & $46(54.0)$ & $63(33.6)$ & 0.122 \\
\hline
\end{tabular}

Values are presented as mean + standard deviation or number $(\%)$

*The sample population for the duration of fever after oseltamivir administration (day) are 14,70, and 130 for $A / H 1 \mathrm{~N} 1$, A/H3N2, and B, respectively. The sample population for the duration of fever in non-oseltaminiir recepients (day) are 30 and 57 for $A / H 3 N 2$ and $B$, respectively.

Table 2. Presentation of Subjective Symptoms according to

Type of Influenza in Children Aged $\geq 24$ Months

\begin{tabular}{lcccc} 
Type of influenza & A/H1N1 $(n=7)$ & A/H3N2 (n=49) & $B(n=146)$ & $P$-value \\
Sore throat & $4(57.1)$ & $26(53.1)$ & $51(35.0)$ & 0.052 \\
\hline Headache & $1(14.3)$ & $19(38.8)$ & $35(24.0)$ & 0.097 \\
\hline Abdominal pain & $1(14.3)$ & $5(10.2)$ & $44(30.1)$ & 0.013 \\
\hline
\end{tabular}

Values are presented as number (\%).

Conclusions: There were significant differences between influenza $A$ and $B$ groups in terms of age, demographics, and clinical course. Although the effectiveness of oseltamivir on influenza differs according to the type of influenza, our data provides evidence that oseltamivir is beneficial for both $A$ and $B$ infections. 